58, 1, pp. 169-182, Warsaw 2020

DOI: $10.15632 /$ jtam-pl/115390

\title{
A SLIDING MODE CONTROLLER DESIGN FOR A MISSILE AUTOPILOT SYSTEM
}

\author{
WiTOLD BuŻANTOWICZ \\ Military University of Technology, Faculty of Mechatronics and Aerospace, Warsaw, Poland \\ e-mail: witold.buzantowicz@wat.edu.pl
}

\begin{abstract}
A description is given of an application of the sliding mode control (SMC) for stabilizing the static and dynamic characteristics of an anti-aircraft missile. The solution provides effective separation of the control process from the dynamics of the missile airframe. In the equivalent part of the stabilization system, a linear-quadratic regulator (LQR) is considered, and an analytical method of selecting the weighting elements of the gain matrix is proposed. This eliminates the need for an iterative solution of the Riccati equation. A nonlinear switching component is introduced into the control signal to provide smoothness of the system response. In simulation tests, the proposed solution was evaluated against selected quantity indices. The paper ends with observations and conclusions.
\end{abstract}

Keywords: anti-aircraft missile, autopilot, sliding mode control, linear-quadratic regulator

\section{Introduction}

Contemporary anti-aircraft missiles offer high maneuverability and continuously improving combat capabilities. During the flight, the physical properties of the missile airframe vary nonlinearly with time and depend on - among other factors - the missile speed and height of the flight. Changes in these properties may also be caused by external interference, which means that the system in question is generally nonstationary and random. In turn, the nonstationary nature of static and dynamic characteristics of the missile airframe determines the changes in characteristics of the entire guidance loop, which in extreme cases may compromise its stability. This is a highly undesirable effect, which has a negative impact on the accuracy of missile guidance to the target. To compensate for the effects of the airframe dynamic characteristics and adverse flight conditions, an autopilot is installed on board the missile as a stabilizing device (Grycewicz et al., 1984; Siouris, 2004; Yanushevsky, 2007; Zarchan, 2012).

Over the past decade or more, the sliding mode control (SMC) has gained in importance as a nonlinear control method which alters the dynamics of the system by means of a discontinuous control signal. SMC algorithms have already been effectively implemented in models of control and guidance systems for anti-aircraft missiles (Zhou et al., 1999; Gu et al., 2005; Shima et al., 2006; Idan et al., 2007; Shtessel and Tournes, 2009).

In the sliding mode controller, the control signal is the sum of the equivalent control and switching control signals. The equivalent control may be provided using any regulator that is able to track the set trajectory with specified accuracy. Here, a linear-quadratic regulator (LQR) will be considered.

The determination of the weighting coefficients of an LQR - algorithmically simple in the case of linear time-invariant (LTI) systems - becomes more complex when the analyzed system contains nonlinear and nonstationary features. In such cases, for solving the Riccati equation to find coefficients of the gain matrix $\mathbf{K}$ in the feedback loop, numerical computation methods are commonly applied to a "frozen" set of coefficients describing the state of the system in a finite 
time horizon, which is made as short as possible. The main difficulty with this approach is the requirement for computations to be carried out in real time (Çimen, 2008; Siddiq et al., 2013) in addition to certain complications related to the application of numerical methods (for instance, bad conditioning of the matrix). It is therefore advantageous to be able to find the form of the weighting coefficients by analytical methods, which eliminate the need for iterative solution of the Riccati equation.

An application of the SMC method for stabilizing the static and dynamic characteristics of an anti-aircraft missile airframe will be presented below. The weighting coefficients of the gain matrix $\mathbf{K}$ of the LQR in the equivalent part are determined here for a linearized system that is subject to a number of simplifications. This enables a solution to be found in a general form. To provide required smoothness of the system response, a nonlinear component of the switching part of the sliding mode controller is introduced into the control signal.

The paper is organized as follows. In Sections 2 and 3, the problem is formulated and a mathematical model of the dynamics of an anti-aircraft missile airframe is presented. In Section 4, a solution is proposed for the equivalent and switching parts of the sliding mode controller for stabilizing static and dynamic characteristics of the airframe for the system in question. Section 5 presents selected results of simulation tests. And the final Section contains observations and conclusions.

\section{Formulation of the problem}

When guiding a missile towards a target, it is desirable that

$$
\omega \approx \dot{\theta}
$$

namely, that the angular rate $\omega$ of the airframe be approximately equal to the angular rate $\dot{\theta}$ of the missile velocity vector, and not depend on the dynamics of the missile itself (Bużantowicz and Pietrasieński, 2018; Grycewicz et al., 1984). Hence, a solution is sought which minimizes the value of the quantity index given in the form of the functional

$$
J=\int_{0}^{t_{f}}|\omega-\dot{\theta}| d t
$$

where $t_{f}$ is the final time of the guidance process. The value of the integral in Eq. (2.2) depends on the ability of the system to reduce the effect of components associated with the dynamics of the missile airframe.

\section{Model of the missile airframe dynamics}

The missile considered here uses a canard-controlled aerodynamic system and roll angle stabilization. The motion of the missile may be considered independently in the control planes.

The differential equations describing the dynamics of the missile motion in the control plane are derived for the scheme shown in Fig. 1. The equation of motion relative to an axis normal to the velocity vector $\mathbf{V}$ is obtained by projecting the forces acting on the missile onto that axis

$$
m V \dot{\theta}=T \sin \alpha+\frac{\rho V^{2}}{2} S c_{L}(\mathrm{Ma}, \alpha)+\frac{\rho V^{2}}{2} S c_{C}(\mathrm{Ma}, \alpha, \delta)
$$

where $T$ is the thrust, $m$ is mass of the missile, $V=|\mathbf{V}|$ is the missile velocity, Ma is the Mach number, $\alpha$ is the airframe angle of attack, $\delta$ is the angle of deflection of the canards, $\rho$ is 


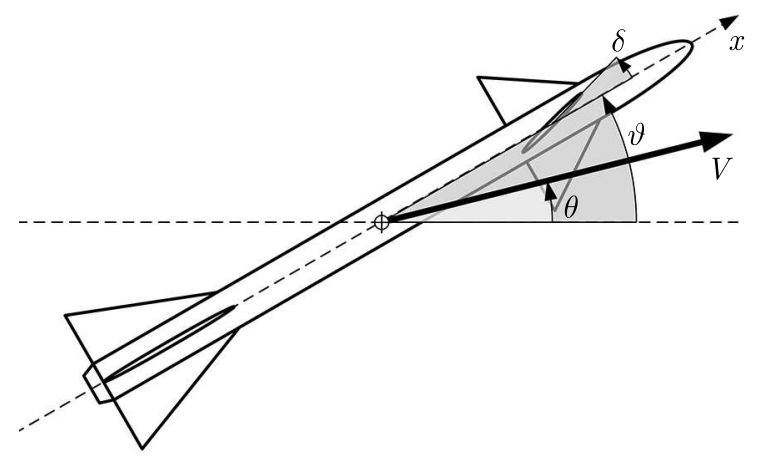

Fig. 1. Angular relationships assumed for the missile in the control plane

atmospheric density, $S$ is the characteristic surface, and $c_{L}$ and $c_{C}$ are aerodynamic coefficients of the airframe and canards. Assuming that the gravitational force acting on the airframe is treated as an external disturbation compensated by a component added to the control signal, Eq. (3.1) may be used to describe the missile dynamics in both the yaw and pitch planes.

The equation of rotational motion is obtained by balancing the moments of forces acting on the missile

$$
I \dot{\omega}=-\frac{\rho V^{2}}{2} S l c_{M}^{\alpha}(\mathrm{Ma}, \alpha)-\frac{\rho V}{2} S l^{2} c_{M}^{\omega}(\mathrm{Ma}, \omega)+\frac{\rho V^{2}}{2} S l c_{M}^{\delta}(\mathrm{Ma}, \alpha, \delta)
$$

where $I$ is the airframe moment of inertia, $\omega$ is the airframe angular rate, $l$ is the characteristic linear dimension, and $c_{M}^{(\cdot)}$ are in general coefficients of aerodynamic moments, particularly, $c_{M}^{\omega}$ is the damping coefficient, $c_{M}^{\alpha}$ is the stabilizing coefficient of the airframe and $c_{M}^{\delta}$ is the control coefficient.

For airframe controller design purposes, taking the coefficients of aerodynamic forces and moments to be linearly dependent on the variables $\alpha, \omega$ and $\delta$, and linearizing the sine function for small values of the angle of attack, a linearized system of equations is obtained to describe the dynamics of the missile airframe in the control plane

$$
\begin{aligned}
& \dot{\theta}=\left\{\frac{\rho V}{2 m} S\left[c_{L}(\mathrm{Ma})+c_{C}(\mathrm{Ma})\right]+\frac{T}{m V}\right\} \alpha+\frac{\rho V}{2 m} S c_{C}(\mathrm{Ma}) \delta \\
& \dot{\omega}=-\left\{\frac{\rho V^{2}}{2 I} S l\left[c_{M}^{\alpha}(\mathrm{Ma})-c_{M}^{\delta}(\mathrm{Ma})\right]\right\} \alpha-\frac{\rho V}{2 I} S l^{2} c_{M}^{\omega}(\mathrm{Ma}) \omega+\frac{\rho V^{2}}{2 I} S l c_{M}^{\delta}(\mathrm{Ma}) \delta
\end{aligned}
$$

Introducing the following notations

$$
\begin{aligned}
& a_{1}=\frac{\rho V}{2 m} S\left[c_{L}(\mathrm{Ma})+c_{C}(\mathrm{Ma})\right]+\frac{T}{m V} \quad a_{2}=\frac{\rho V^{2}}{2 I} S l\left[c_{M}^{\alpha}(\mathrm{Ma})-c_{M}^{\delta}(\mathrm{Ma})\right] \\
& a_{3}=\frac{\rho V}{2 I} S l^{2} c_{M}^{\omega}(\mathrm{Ma}) \quad b_{1}=\frac{\rho V}{2 m} S c_{C}(\mathrm{Ma}) \quad b_{2}=\frac{\rho V^{2}}{2 I} S l c_{M}^{\delta}(\mathrm{Ma})
\end{aligned}
$$

the system of equations (3.3) takes the form

$$
\dot{\theta}=a_{1} \alpha+b_{1} \delta \quad \dot{\omega}=-a_{2} \alpha-a_{3} \omega+b_{2} \delta
$$

Equations in (3.5) are supplemented by the following relationships

$$
\vartheta=\int \omega d t \quad a=V \dot{\theta} \quad \alpha=\vartheta-\theta
$$

where $\vartheta$ is the pitch (yaw) angle of the airframe and $a$ is the normal acceleration in the control plane, and by the equation modelling the operation of the fin actuators

$$
\dot{\delta}=\frac{\delta_{c}-\delta}{\tau}
$$


where $\delta_{c}$ is the commanded canard deflection angle, $\delta$ is the current canard deflection angle, and $\tau$ is the time constant of servos.

\section{Design of the stabilization system}

The control signal $\kappa_{s m c}$ is the sum of the signals of the equivalent control $\kappa_{e q}$ and switching control $\kappa_{s w}$

$$
\kappa_{s m c}=\kappa_{e q}+\kappa_{s w}
$$

Synthesis of the switching control is associated with the design of a stable sliding surface $\sigma$, while synthesis of the equivalent control involves design of a control process which will bring the states of the system onto a selected sliding surface in a finite time.

For the provision of equivalent control, a linear-quadratic regulator (LQR) is selected. For synthesis of its parameters, the linearized model of the missile motion described by the system of equations (3.5) is used. Noting that

$$
\alpha=\vartheta-\theta \quad \rightarrow \quad \dot{\alpha}=\dot{\vartheta}-\dot{\theta}=\omega-\dot{\theta}
$$

we obtain the following equivalent form of the system of equations (3.5)

$$
\dot{\alpha}=-a_{1} \alpha+\omega-b_{1} \delta \quad \dot{\omega}=-a_{2} \alpha-a_{3} \omega+b_{2} \delta
$$

Switching to vector-matrix notation, we have

$$
\left[\begin{array}{c}
\dot{\alpha} \\
\dot{\omega}
\end{array}\right]=\left[\begin{array}{cc}
-a_{1} & 1 \\
-a_{2} & -a_{3}
\end{array}\right]\left[\begin{array}{c}
\alpha \\
\omega
\end{array}\right]+\left[\begin{array}{c}
-b_{1} \\
b_{2}
\end{array}\right] \delta \quad \omega_{\text {out }}=\left[\begin{array}{ll}
0 & 1
\end{array}\right]\left[\begin{array}{c}
\alpha \\
\omega
\end{array}\right]+[0] \delta
$$

or more concisely

$$
\dot{\mathbf{x}}=\mathbf{A x}+\mathbf{B} \delta \quad y=\mathbf{C x}+D \delta
$$

To simplify further considerations, the inertia of the fin actuator is ignored in the equivalent part of the stabilization system; that is, it is assumed that $\kappa=\delta_{c}=\delta$.

The regulator settings are determined based on the linear dynamic equations and a quadratic cost function in the form

$$
J_{L Q}=\int_{0}^{\infty}\left[\mathbf{x}^{\mathrm{T}}(t) \mathbf{Q} \mathbf{x}(t)+\boldsymbol{\kappa}^{\mathrm{T}}(t) \mathbf{R} \boldsymbol{\kappa}(t)\right] d t
$$

where $\mathbf{Q}=\mathbf{Q}^{\mathrm{T}}$ and $\mathbf{R}=\mathbf{R}^{\mathrm{T}}$ are weighting parameters for the state variable signals and the control signals. The values of these parameters are selected arbitrarily.

To determine the gain matrix $\mathbf{K}$ of the equivalent part

$$
\kappa_{e q}=N \kappa-\mathbf{K} \mathbf{x}=N \kappa-\mathbf{R}^{-1} \mathbf{B}^{\mathrm{T}} \mathbf{P} \mathbf{x}
$$

where $N$ is the scaling factor of the input signal $\kappa$, it is necessary to find the matrix $\mathbf{P}$ satisfying the Riccati equation

$$
\mathbf{A}^{\mathrm{T}} \mathbf{P}+\mathbf{P A}-\mathbf{P B R}^{-1} \mathbf{B}^{\mathrm{T}} \mathbf{P}+\mathbf{Q}=\mathbf{0}
$$

In the general case, it is not possible to determine the entries of $\mathbf{P}$ analytically (Çimen, 2008). However, in certain situations, their form can be obtained, and this makes it possible to define 
an adaptable feedback from the time-variable state of the system. We take the matrices $\mathbf{P}, \mathbf{Q}$ and $\mathbf{R}$ to be

$$
\mathbf{P}=\left[\begin{array}{ll}
p_{11} & p_{12} \\
p_{21} & p_{22}
\end{array}\right] \quad \mathbf{Q}=\left[\begin{array}{cc}
q_{1} & 0 \\
0 & q_{2}
\end{array}\right] \quad \mathbf{R}=[r]
$$

hence the gain matrix $\mathbf{K}$ takes the form

$$
\mathbf{K}=\frac{1}{r}\left[\begin{array}{ll}
-b_{1} & b_{2}
\end{array}\right]\left[\begin{array}{ll}
p_{11} & p_{12} \\
p_{21} & p_{22}
\end{array}\right]=\frac{1}{r}\left[\begin{array}{ll}
-b_{1} p_{11}+b_{2} p_{21} & -b_{1} p_{12}+b_{2} p_{22}
\end{array}\right]
$$

The expanded form of Eq. (4.8) is as follows

$$
\begin{aligned}
& {\left[\begin{array}{cc}
-a_{1} & -a_{2} \\
1 & -a_{3}
\end{array}\right]\left[\begin{array}{ll}
p_{11} & p_{12} \\
p_{21} & p_{22}
\end{array}\right]+\left[\begin{array}{ll}
p_{11} & p_{12} \\
p_{21} & p_{22}
\end{array}\right]\left[\begin{array}{cc}
-a_{1} & 1 \\
-a_{2} & -a_{3}
\end{array}\right]} \\
& -\left\{\left[\begin{array}{ll}
p_{11} & p_{12} \\
p_{21} & p_{22}
\end{array}\right]\left[\begin{array}{c}
-b_{1} \\
b_{2}
\end{array}\right]\right\} \frac{1}{r}\left\{\left[\begin{array}{ll}
-b_{1} & b_{2}
\end{array}\right]\left[\begin{array}{ll}
p_{11} & p_{12} \\
p_{21} & p_{22}
\end{array}\right]\right\}+\left[\begin{array}{cc}
q_{1} & 0 \\
0 & q_{2}
\end{array}\right]=\left[\begin{array}{ll}
0 & 0 \\
0 & 0
\end{array}\right]
\end{aligned}
$$

and

$$
\begin{aligned}
& {\left[\begin{array}{cc}
-a_{1} p_{11}-a_{2} p_{21} & -a_{1} p_{12}-a_{2} p_{22} \\
p_{11}-a_{3} p_{21} & p_{12}-a_{3} p_{22}
\end{array}\right]+\left[\begin{array}{cc}
-a_{1} p_{11}-a_{2} p_{12} & p_{11}-a_{3} p_{12} \\
-a_{1} p_{21}-a_{2} p_{22} & p_{21}-a_{3} p_{22}
\end{array}\right]} \\
& \quad-\frac{1}{r}\left[\begin{array}{cc}
b_{1}^{2} p_{11}^{2}-b_{1} b_{2} p_{11} p_{12}-b_{1} b_{2} p_{11} p_{21}+b_{2}^{2} p_{12} p_{21} & b_{1}^{2} p_{11} p_{12}-b_{1} b_{2} p_{11} p_{22}-b_{1} b_{2} p_{12}^{2}+b_{2}^{2} p_{12} p_{22} \\
b_{1}^{2} p_{11} p_{21}-b_{1} b_{2} p_{21}^{2}-b_{1} b_{2} p_{11} p_{22}+b_{2}^{2} p_{21} p_{22} & b_{1}^{2} p_{12} p_{21}-b_{1} b_{2} p_{21} p_{22}-b_{1} b_{2} p_{12} p_{22}+b_{2}^{2} p_{22}^{2}
\end{array}\right] \\
& \quad+\left[\begin{array}{cc}
q_{1} & 0 \\
0 & q_{2}
\end{array}\right]=\left[\begin{array}{ll}
0 & 0 \\
0 & 0
\end{array}\right]
\end{aligned}
$$

As the lift on the airframe and wings in fin-controlled missiles is many times greater than the lift on the fins (Grycewicz et al., 1984), it may be assumed without introducing a significant error that

$$
a_{1} \gg b_{1} \quad \rightarrow \quad b_{1}=0
$$

In this case, Eq. (4.12) reduces to the form

$$
\begin{aligned}
& {\left[\begin{array}{cc}
-a_{1} p_{11}-a_{2} p_{21} & -a_{1} p_{12}-a_{2} p_{22} \\
p_{11}-a_{3} p_{21} & p_{12}-a_{3} p_{22}
\end{array}\right]+\left[\begin{array}{ll}
-a_{1} p_{11}-a_{2} p_{12} & p_{11}-a_{3} p_{12} \\
-a_{1} p_{21}-a_{2} p_{22} & p_{21}-a_{3} p_{22}
\end{array}\right]} \\
& -\frac{b_{2}^{2}}{r}\left[\begin{array}{ll}
p_{12} p_{21} & p_{12} p_{22} \\
p_{21} p_{22} & p_{22} p_{22}
\end{array}\right]+\left[\begin{array}{cc}
q_{1} & 0 \\
0 & q_{2}
\end{array}\right]=\left[\begin{array}{ll}
0 & 0 \\
0 & 0
\end{array}\right]
\end{aligned}
$$

and the gain matrix $\mathbf{K}$ is equal to

$$
\mathbf{K}=\frac{1}{r}\left[\begin{array}{ll}
0 & b_{2}
\end{array}\right]\left[\begin{array}{ll}
p_{11} & p_{12} \\
p_{21} & p_{22}
\end{array}\right]=\frac{b_{2}}{r}\left[\begin{array}{ll}
p_{21} & p_{22}
\end{array}\right]
$$

This means that to determine the settings of the LQR it is sufficient to find just two of the four entries of the matrix $\mathbf{P}$.

By summation of the matrices in Eq. (4.14), and comparison of the corresponding terms and rearrangement, the following system of equations is obtained

$$
\begin{array}{lc}
-2 a_{1} p_{11}-a_{2} p_{12}-a_{2} p_{21}+q_{1}=\frac{b_{2}^{2}}{r} p_{12} p_{21} & p_{11}-\left(a_{1}+a_{3}\right) p_{12}-a_{2} p_{22}=\frac{b_{2}^{2}}{r} p_{12} p_{22} \\
p_{11}-\left(a_{1}+a_{3}\right) p_{21}-a_{2} p_{22}=\frac{b_{2}^{2}}{r} p_{21} p_{22} & p_{12}+p_{21}-2 a_{3} p_{22}+q_{2}=\frac{b_{2}^{2}}{r} p_{22} p_{22}
\end{array}
$$


From the second and third equations in (4.16), we have

$$
\left(p_{21}-p_{12}\right)\left(a_{1}+a_{3}+\frac{b_{2}^{2}}{r} p_{22}\right)=0 \quad \rightarrow \quad p_{12} \equiv p_{21}
$$

Thus, the system of equations (4.16) simplifies to

$$
\begin{aligned}
& -2 a_{1} p_{11}-2 a_{2} p_{21}+q_{1}=\frac{b_{2}^{2}}{r} p_{21} p_{21} \quad p_{11}-\left(a_{1}+a_{3}\right) p_{21}-a_{2} p_{22}=\frac{b_{2}^{2}}{r} p_{21} p_{22} \\
& 2 p_{21}-2 a_{3} p_{22}+q_{2}=\frac{b_{2}^{2}}{r} p_{22} p_{22}
\end{aligned}
$$

Multiplying both sides of the second equation in (4.18) by 2, and summing the equations in (4.18), we obtain

$$
\frac{2 r}{b_{2}^{2}}\left(1-a_{1}\right) p_{11}+\frac{2 r}{b_{2}^{2}}\left(1-a_{1}-a_{2}-a_{3}\right) p_{21}+\frac{2 r}{b_{2}^{2}}\left(-a_{2}-a_{3}\right) p_{22}=\left(p_{21}+p_{22}\right)^{2}-\frac{r}{b_{2}^{2}}\left(q_{1}+q_{2}\right)
$$

Assuming that

$$
\left(p_{21}+p_{22}\right)^{2}-\frac{r}{b_{2}^{2}}\left(q_{1}+q_{2}\right)=0
$$

and choosing $r, q_{1}$ and $q_{2}$ such that (Erdem and Alleyne, 2004; Çimen, 2008)

$$
r>0, \quad q>0 \wedge q=q_{1}=q_{2}
$$

we have

$$
\left(p_{21}+p_{22}\right)^{2}=\frac{2 r}{b_{2}^{2}} q
$$

Because $r>0$ and $q>0$, it follows that

$$
p_{21}+p_{22}= \pm \sqrt{\frac{2 r}{b_{2}^{2}} q}
$$

The expression under the root sign in (4.23) is real and positive, and thus has a square root in the set of positive real numbers. Assuming additionally $r=2 q$, two possible solutions are obtained

$$
p_{21}=\frac{r}{b_{2}}-p_{22} \quad p_{21}=-\frac{r}{b_{2}}-p_{22}
$$

Solving the first equation in (4.18) with respect to $p_{21}$ is inconvenient because of the complex substitution for the entry $p_{11}$

$$
p_{11}=\frac{b_{2}^{2}}{r} p_{21} p_{22}+\left(a_{1}+a_{3}\right) p_{21}+a_{2} p_{22}
$$

however, after some transformations, by Eq. (4.15), four pairs of entries for the matrix $\mathbf{K}$ can be obtained

$$
\begin{aligned}
& k_{1}=-\frac{1}{b_{2}\left(1-2 a_{1}\right)}\left[a_{1}\left(a_{1}-a_{2}+a_{3}+b_{2}\right)+a_{2} \pm \sqrt{\Psi_{1}}\right] \\
& k_{2}=1+\frac{1}{b_{2}\left(1-2 a_{1}\right)}\left[a_{1}\left(a_{1}-a_{2}+a_{3}+b_{2}\right)+a_{2} \pm \sqrt{\Psi_{1}}\right]
\end{aligned}
$$


and

$$
\begin{aligned}
& k_{1}=-\frac{1}{b_{2}\left(1-2 a_{1}\right)}\left[a_{1}\left(a_{1}-a_{2}+a_{3}-b_{2}\right)+a_{2} \pm \sqrt{\Psi_{2}}\right] \\
& k_{2}=-1+\frac{1}{b_{2}\left(1-2 a_{1}\right)}\left[a_{1}\left(a_{1}-a_{2}+a_{3}-b_{2}\right)+a_{2} \pm \sqrt{\Psi_{2}}\right]
\end{aligned}
$$

where $\Psi_{1}$ and $\Psi_{2}$ are given as follows

$$
\begin{aligned}
\Psi_{1} & =\left(a_{1} b_{2}+a_{2}\right)^{2}+\left[a_{1}\left(a_{1}+a_{3}\right)-a_{1} a_{2}\right]^{2}+2\left(a_{1}-a_{2}+a_{3}\right)\left(a_{1}^{2} b_{2}+a_{1} a_{2}\right) \\
& +\left(\frac{1}{2}-a_{1}\right)\left(b_{2}^{2}-4 a_{1} a_{2} b_{2}\right) \\
\Psi_{2} & =\left(a_{1} b_{2}-a_{2}\right)^{2}+\left[a_{1}\left(a_{1}+a_{3}\right)-a_{1} a_{2}\right]^{2}-2\left(a_{1}-a_{2}+a_{3}\right)\left(a_{1}^{2} b_{2}-a_{1} a_{2}\right) \\
& +\left(\frac{1}{2}-a_{1}\right)\left(b_{2}^{2}+4 a_{1} a_{2} b_{2}\right)
\end{aligned}
$$

Solving the third equation in (4.18) with respect to $p_{22}$ for the cases given by Eq. (4.24), and substituting the achieved results to Eq. (4.15), we obtain four other pairs of entries for the matrix $\mathbf{K}$

$$
\begin{aligned}
& k_{1}=1+\frac{1}{b_{2}}\left[\left(a_{3}+1\right) \mp \sqrt{a_{3}^{2}+2 a_{3}+\frac{1}{2} b_{2}^{2}+2 b_{2}+1}\right] \\
& k_{2}=-\frac{1}{b_{2}}\left[\left(a_{3}+1\right) \pm \sqrt{a_{3}^{2}+2 a_{3}+\frac{1}{2} b_{2}^{2}+2 b_{2}+1}\right]
\end{aligned}
$$

and

$$
\begin{aligned}
& k_{1}=-1+\frac{1}{b_{2}}\left[\left(a_{3}+1\right) \mp \sqrt{a_{3}^{2}+2 a_{3}+\frac{1}{2} b_{2}^{2}-2 b_{2}+1}\right] \\
& k_{2}=-\frac{1}{b_{2}}\left[\left(a_{3}+1\right) \pm \sqrt{a_{3}^{2}+2 a_{3}+\frac{1}{2} b_{2}^{2}-2 b_{2}+1}\right]
\end{aligned}
$$

The three pairs of those defined in (4.26), (4.27) and (4.29), (4.30) give unstable solutions (with positive feedback), and the other four lead to inappropriate quality of the system response (Fig. 2a). These solutions should be rejected. Based on the analysis of the angular rate $\omega$ obtained as step responses of the airframe to the commanded fin deflections, in our further considerations the gain matrix $\mathbf{K}$ will be taken to have the form

$$
\mathbf{K}=\left[\begin{array}{l}
k_{1} \\
k_{2}
\end{array}\right]^{\mathrm{T}}=\left[\begin{array}{c}
-\frac{1}{b_{2}\left(1-2 a_{1}\right)}\left[a_{1}\left(a_{1}-a_{2}+a_{3}-b_{2}\right)+a_{2}+\sqrt{\Psi}\right] \\
-1+\frac{1}{b_{2}\left(1-2 a_{1}\right)}\left[a_{1}\left(a_{1}-a_{2}+a_{3}-b_{2}\right)+a_{2}+\sqrt{\Psi}\right]
\end{array}\right]^{\mathrm{T}}
$$

with $\Psi=\Psi_{2}$ as it is defined by Eq. (4.28) 2 .

The full-state feedback system does not compare the output to the reference. Instead, it compares the state vector $\mathbf{x}$ multiplied by the gain matrix $\mathbf{K}$ to the reference. To obtain the desired output, we need to scale the reference input so that the output equals the reference. The feed-forwarding scaling factor of the input signal is taken to be

$$
N=N_{\kappa}+\mathbf{K N}_{x}
$$

where

$$
\left[\begin{array}{l}
\mathbf{N}_{x} \\
N_{\kappa}
\end{array}\right]=\boldsymbol{\Omega}^{-1}\left[\begin{array}{l}
\mathbf{0} \\
\zeta
\end{array}\right] \quad \boldsymbol{\Omega}=\left[\begin{array}{ll}
\mathbf{A} & \mathbf{B} \\
\mathbf{C} & D
\end{array}\right]=\left[\begin{array}{ccc}
-a_{1} & 1 & -b_{1} \\
-a_{2} & -a_{3} & b_{2} \\
0 & 1 & 0
\end{array}\right]
$$


(a)

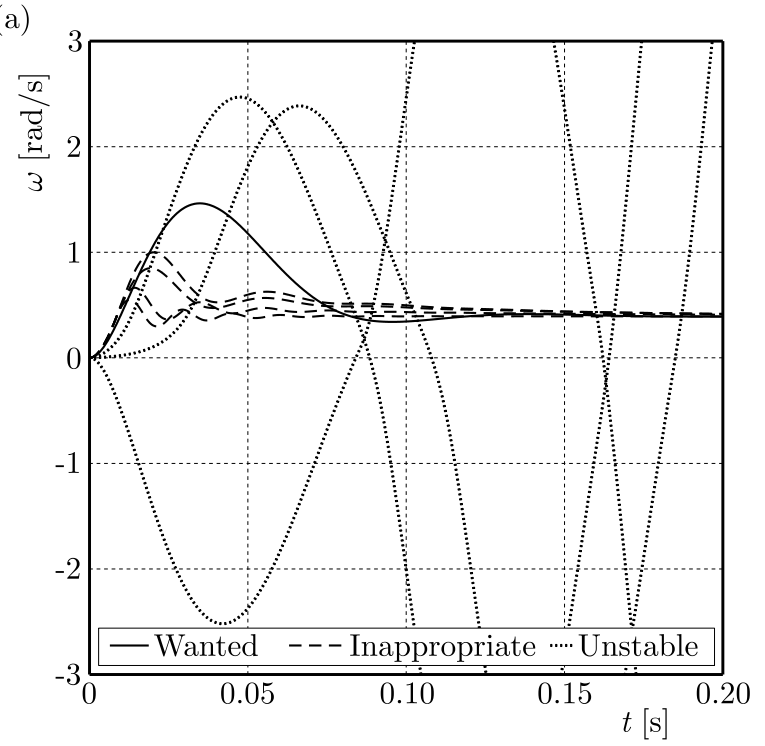

(b)

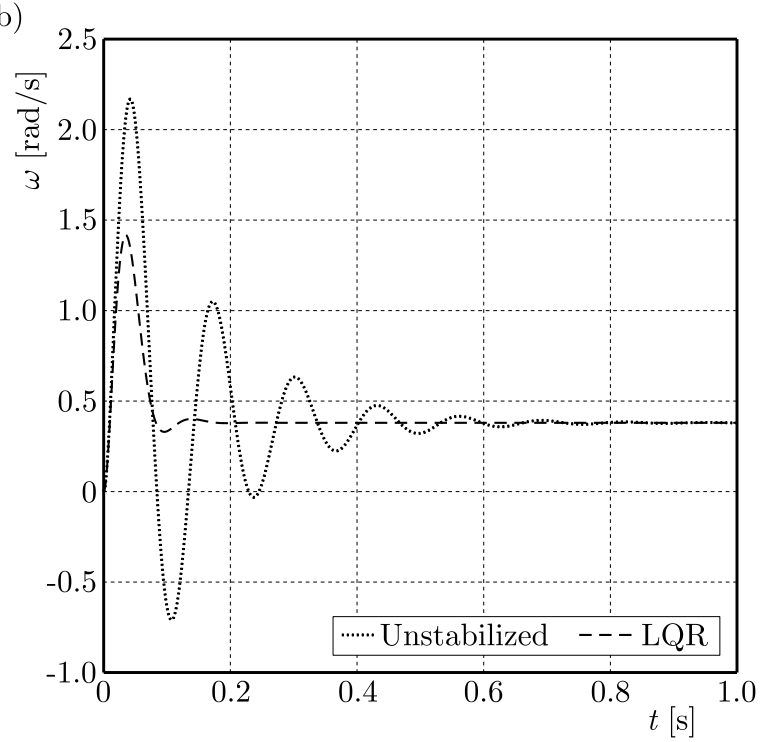

Fig. 2. Angular rates of the airframe with the LQR stabilization system for the derived pairs of entries of the gain matrix $\mathbf{K}$ (a) and angular rates of the unstabilized airframe and of the airframe having a stabilization system with the chosen linear-quadratic regulator (b), obtained as step responses for the commanded fin deflections

and $\zeta$ is the factor required to compensate the steady-state error

$$
\zeta=\frac{a_{1} b_{2}+a_{2} b_{1}}{a_{1} a_{3}+a_{2}}
$$

calculated for $t=0$ and treated as a constant value in a finite time horizon.

The determinant of the matrix $\boldsymbol{\Omega}$ is non-zero

$$
\operatorname{det} \boldsymbol{\Omega}=a_{1} b_{2}+a_{2} b_{1}
$$

which ensures the existence of an inverse matrix. The inverse matrix $\Omega^{-1}$ is given by

$$
\boldsymbol{\Omega}^{-1}=\frac{1}{\operatorname{det} \boldsymbol{\Omega}}\left(\boldsymbol{\Omega}_{D}\right)^{\mathrm{T}}=\left[\begin{array}{ccc}
\frac{-b_{2}}{a_{1} b_{2}+a_{2} b_{1}} & \frac{-b_{1}}{a_{1} b_{2}+a_{2} b_{1}} & \frac{-a_{3} b_{1}+b_{2}}{a_{1} b_{2}+a_{2} b_{1}} \\
0 & 0 & 1 \\
\frac{-a_{2}}{a_{1} b_{2}+a_{2} b_{1}} & \frac{a_{1}}{a_{1} b_{2}+a_{2} b_{1}} & \frac{a_{1} a_{3}+a_{2}}{a_{1} b_{2}+a_{2} b_{1}}
\end{array}\right]
$$

Now

$$
\mathbf{N}_{x}=\left[\begin{array}{c}
\zeta\left(\frac{-a_{3} b_{1}+b_{2}}{a_{1} b_{2}+a_{2} b_{1}}\right) \\
\zeta
\end{array}\right] \quad N_{\kappa}=\zeta\left(\frac{a_{1} a_{3}+a_{2}}{a_{1} b_{2}+a_{2} b_{1}}\right)
$$

and by Eqs. (4.31) and (4.32), the scaling factor of the input signal $\kappa$ takes the form

$$
N=\frac{\zeta}{a_{1} b_{2}+a_{2} b_{1}}\left[a_{1} a_{3}+a_{2}+k_{1}\left(-a_{3} b_{1}+b_{2}\right)+k_{2}\left(a_{1} b_{2}+a_{2} b_{1}\right)\right]
$$

while the control law of the equivalent part of the stabilization system is given by

$$
\kappa_{e q}=N \kappa-k_{1} \alpha-k_{2} \omega
$$

Figure $2 \mathrm{~b}$ presents angular rate histories for both the unstabilized airframe and the airframe with a stabilization system composed exclusively of the equivalent part with the LQR. 
In the design of the switching part of the stabilization system, it is assumed that the derivative of the angular rate of the airframe is conditioned by some function $f$ of $\omega$ and by the control signal $\kappa$

$$
\dot{\omega}=f(\omega)+\kappa
$$

where at any selected time instant only an estimate $\omega_{e}$ of the missile angular rate $\omega$ is known. Hence

$$
f(\omega)-f\left(\omega_{e}\right)=\varepsilon
$$

In the ideal case, the difference $\varepsilon$ is equal to zero. It is assumed, moreover, that the difference between the values of $f(\omega)$ and $f\left(\omega_{e}\right)$ is bounded

$$
\left|f(\omega)-f\left(\omega_{e}\right)\right| \leqslant \varepsilon_{\max }
$$

Taking the above into account, the sliding surface $\sigma=0$ is defined using the variable

$$
\sigma=\zeta^{-1} \omega-\kappa
$$

where $\zeta>0$ is a constant defined by Eq. (4.34). The Lyapunov function candidate has the form

$$
\ell=\frac{1}{2} \sigma^{2}
$$

and its time derivative is

$$
\dot{\ell}=\sigma \dot{\sigma}=\sigma\left(\zeta^{-1} \dot{\omega}-\dot{\kappa}\right)
$$

Because

$$
\dot{\sigma}=\zeta^{-1} \dot{\omega}-\dot{\kappa}=\zeta^{-1}[f(\omega)+\kappa]-\dot{\kappa}
$$

assuming that

$$
\dot{\kappa}=\zeta^{-1}\left[f\left(\omega_{e}\right)+\kappa_{e q}\right] \quad f(\omega)=f\left(\omega_{e}\right) \quad \dot{\sigma}=0
$$

the control law is defined as having the form

$$
\kappa=\kappa_{e q}-k_{3} \operatorname{sgn}(\sigma)
$$

where $\kappa_{e q}$ is the signal of the equivalent part. For the case under consideration

$$
\dot{\ell}=\sigma \dot{\sigma}=\sigma\left(\zeta^{-1} \dot{\omega}-\dot{\kappa}\right)=\sigma\left\{\zeta^{-1}[f(\omega)+\kappa]-\dot{\kappa}\right\}
$$

which, by Eqs. (4.47) and (4.48), gives

$$
\begin{aligned}
& \dot{\ell}=\zeta^{-1} \sigma\left[f(\omega)+\kappa_{e q}-k_{3} \operatorname{sgn}(\sigma)-f\left(\omega_{e}\right)-\kappa_{e q}\right] \\
& \dot{\ell}=-\zeta^{-1} \sigma\left[k_{3} \operatorname{sgn}(\sigma)-\varepsilon_{\max }\right] \quad \dot{\ell}=-\zeta^{-1}|\sigma|\left(k_{3}-\varepsilon_{\max }\right)
\end{aligned}
$$

The assumption

$$
k_{3}>\varepsilon_{\max }
$$

causes the function $\ell$ to be negative, which ensures that the state $\sigma=0$ will be reached in a finite time. To ensure the stability of the switching control based on the Lyapunov theorem, the 
chattering effect for the discontinuous function in Eq. (4.48) has been reduced by replacement with the hyperbolic tangent function

$$
\kappa_{s w}=-k_{3} \tanh \frac{\sigma}{\xi}
$$

where $\xi=2$ is the boundary layer thickness, and $k_{3}=0.25$ is the gain factor of the switching part. The values of the parameters $\xi$ and $k_{3}$ are chosen experimentally.

By Eqs. (4.39) and (4.52), in Eq. (4.1) the control signal for the developed algorithm finally takes the form

$$
\kappa_{s m c}=N \kappa-k_{1} \alpha-k_{2} \omega-k_{3} \tanh \left(\frac{\zeta^{-1} \omega-\kappa}{\xi}\right)
$$

\section{Performance analysis}

The designed stabilization system has been evaluated against the quantity indices defined based on the step responses: of the regulation time $t_{r e g}$ measured according to the angular rate $\omega$ for accuracy ranges $\Delta$ equal to $2 \%$ and $5 \%$, and of the steady-state error $\varepsilon_{r e g}$. In addition, based on a series of the recorded values for the airframe angular rate $\omega$, the angular rate $\dot{\theta}$ of the velocity vector $\mathbf{V}$ and the canard deflection angle $\delta$ were determined for the following quantity indices

$$
J_{\Omega}=\int_{0}^{t_{f}}|\omega-\dot{\theta}| d t \quad J_{\Delta}=\int_{0}^{t_{f}}|\dot{\delta}| d t
$$

where $t_{f}$ is the final time of the simulation.

It is assumed that the missile has no thrust during the tests. The following parameters were assumed in the simulations: $m=100 \mathrm{~kg}, I=35 \mathrm{~kg} \cdot \mathrm{m}^{2}, S=0.67 \mathrm{~m}^{2}, l=1.36 \mathrm{~m}$, $\tau=0.01 \mathrm{~s},|\delta| \leqslant 0.35 \mathrm{rad}$. Values of geometrical and mass coefficients and of aerodynamic forces and moments required for Eqs. (3.4) were determined analytically (Kurow and Dołżanski, 1964). For the derivation of approximating differential equations for the elements of the studied systems, the fourth-order Runge-Kutta numerical integration method was used.

Scenario 1. Figures 3 and 4 and Table 1 give the results obtained from a simulation test of a stabilization system with constant coefficients $a_{1}=5 \mathrm{~s}^{-1}, a_{2}=2350 \mathrm{~s}^{-2}, a_{3}=10 \mathrm{~s}^{-1}$, $b_{1}=0.4 \mathrm{~s}^{-1}, b_{2}=420 \mathrm{~s}^{-2}$ and flight velocity $V=950 \mathrm{~m} / \mathrm{s}$. The step response of the missile airframe was investigated for three cases: a) unstabilized airframe; b) airframe with the LQR stabilization system; and c) airframe stabilized using the SMC system. The time of the simulation was $t_{f}=1 \mathrm{~s}$ (for visualization purposes, the time axis in Figs. 3 and 4 is limited to $0.5 \mathrm{~s}$ ).

Table 1. Values of control quantity indices

\begin{tabular}{|c|c|c|c|c|c|}
\hline \multirow{2}{*}{ Airframe } & \multirow{2}{*}{$\varepsilon_{\text {reg }}[\%]$} & \multicolumn{2}{|c|}{$t_{\text {reg }}[\mathrm{s}]$} & \multirow{2}{*}{$J_{\Omega}[-]$} & \multirow{2}{*}{$J_{\Delta}[-]$} \\
\cline { 3 - 4 } & & $\Delta=2 \%$ & $\Delta=5 \%$ & & \\
\hline \hline Unstabilized & - & 0.76 & 0.64 & $1.995 \mathrm{e} 3$ & $2.955 \mathrm{e} 3$ \\
\hline LQR & 0 & 0.16 & 0.15 & $0.541 \mathrm{e} 3$ & $3.536 \mathrm{e} 3$ \\
\hline SMC & 0 & 0.20 & 0.16 & $0.520 \mathrm{e} 3$ & $4.336 \mathrm{e} 3$ \\
\hline
\end{tabular}

The regulation time is reduced by three-quarters for both the LQR and the SMC stabilization system, and for both, the steady-state error $\varepsilon_{r e g}$ is equal to zero. The lowest value of the quantity index $J_{\Omega}$ was achieved for the SMC system, which confirms the ability of that system to limit effectively the impact exerted on the control process by factors related to the dynamics of the 
(a)

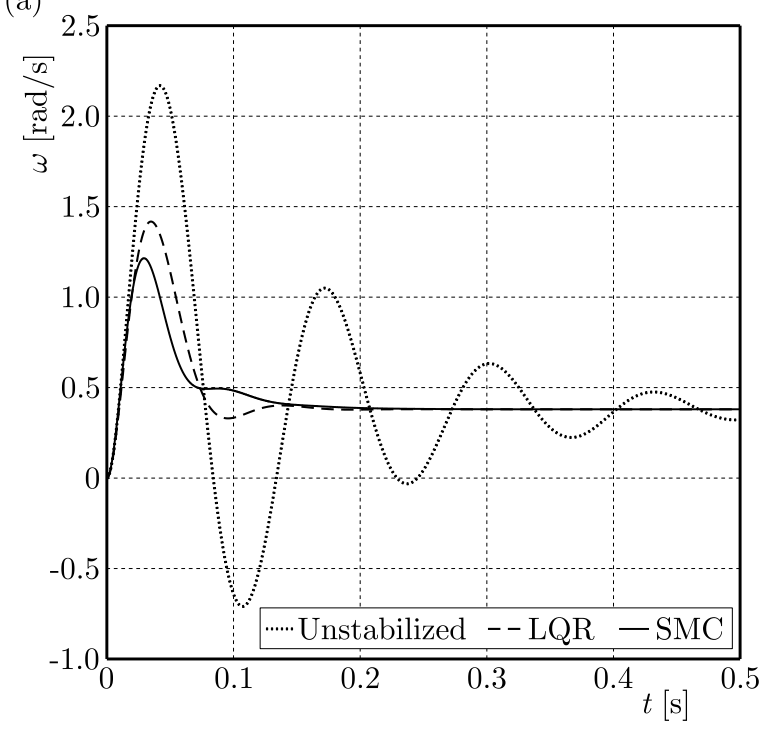

(b)

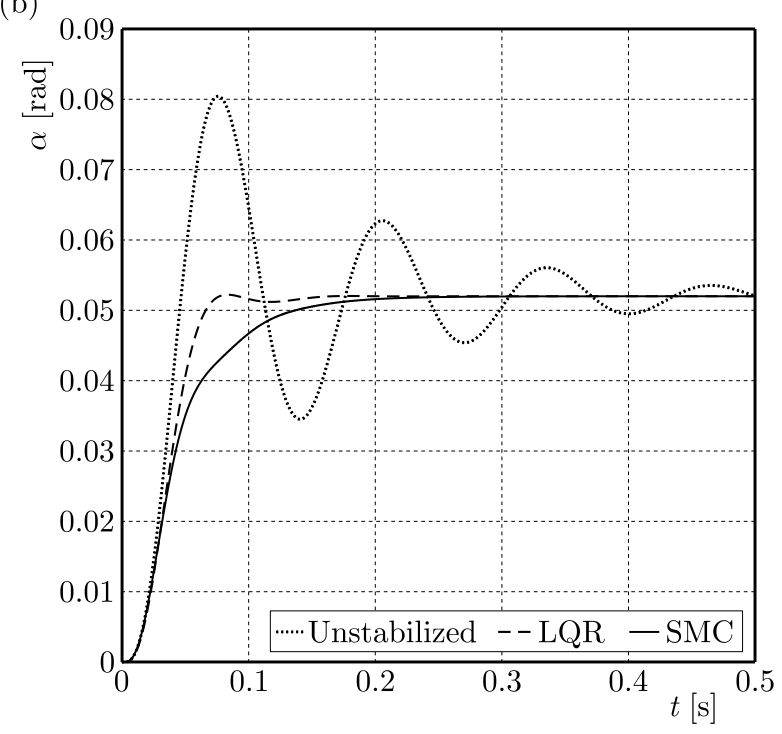

Fig. 3. (a)Angular rates of the airframe. (b) Airframe angles of attack

(a)

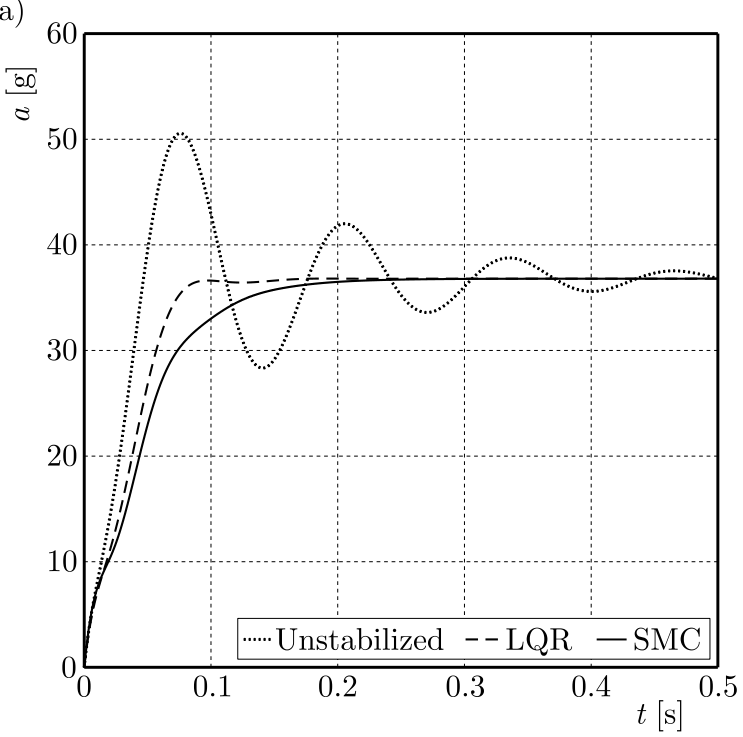

(b)

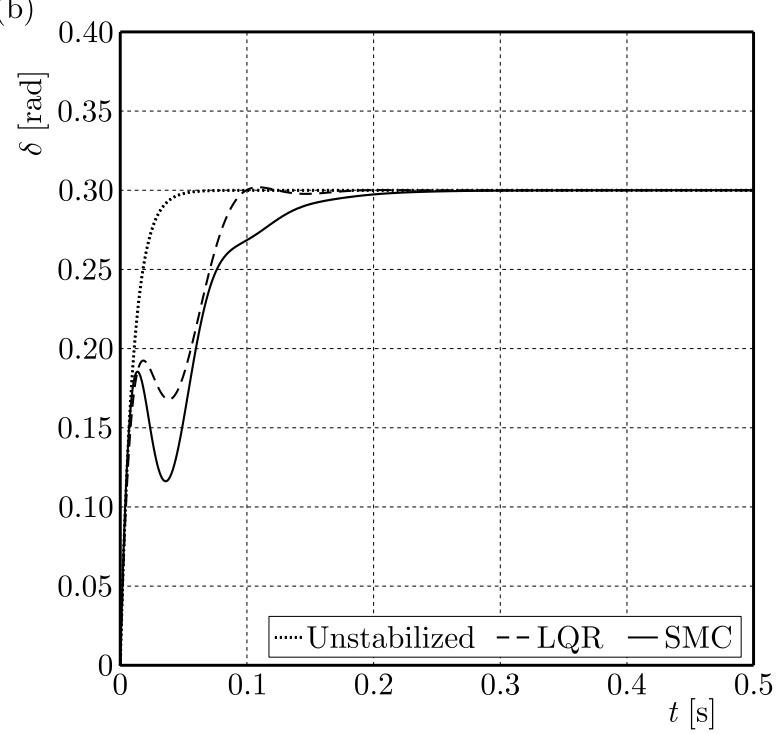

Fig. 4. (a) Normal accelerations of the airframe. (b) Canard deflection angles

missile airframe. However, as expected, the quantity index $J_{\Delta}$ took a higher value for the SMC system than for the LQR, which means that the use of the SMC algorithm in the autopilot is energetically exhausting, as it requires more energy to be delivered to the actuators (the energy cost is directly related to the change in the canard deflection angle, namely to its derivative $\dot{\delta}$ ).

Scenario 2. The results of simulation tests for time-variable coefficients (Fig. 5) are presented in Figs. 6-10 and Table 2. As above, the step response of the missile airframe was investigated for three cases: a) unstabilized airframe; b) airframe with the LQR stabilization system; and c) airframe stabilized by the SMC system. The simulation time was $t_{f}=2 \mathrm{~s}$.

The results obtained for variable coefficients reflect the essence of the operation of the stabilization system the fundamental task of which is to make the value of the output signal independent of the dynamic characteristics of the airframe. This is illustrated by the series of angular rate values shown in Figs. 6 and 7. In the case of the unstabilized airframe, the changes in the values of the parameters describing the dynamics of the missile cause the angular rate $\omega$ 


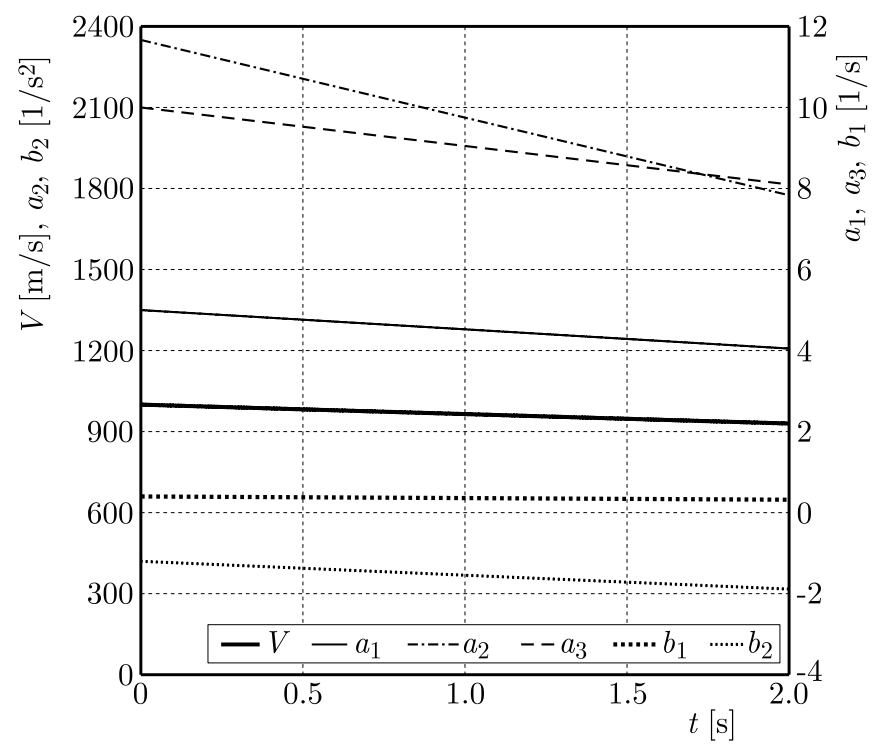

Fig. 5. Changes in the coefficients of Eq. (3.5)

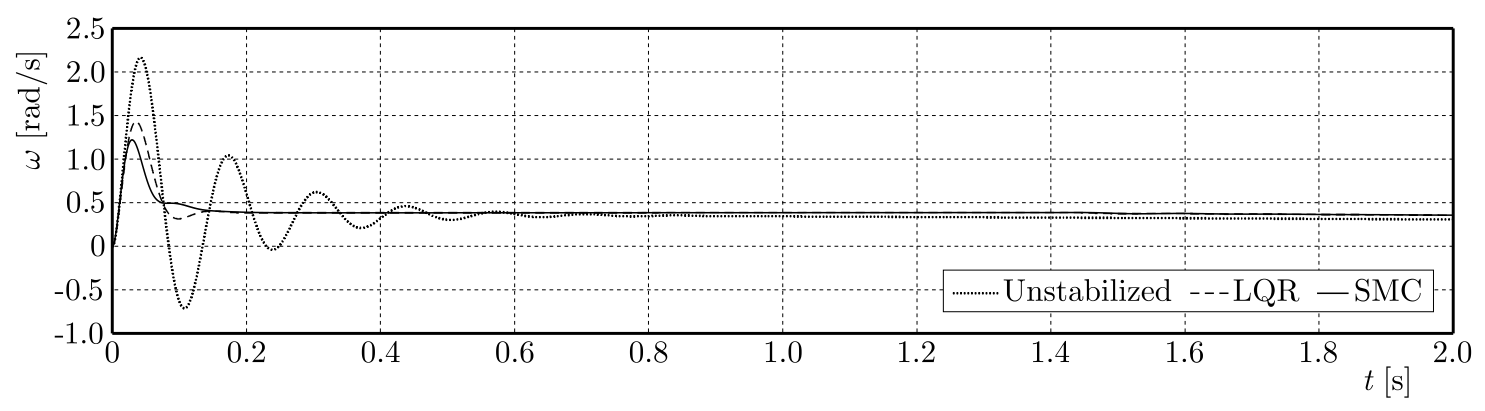

Fig. 6. Angular rates of the airframe

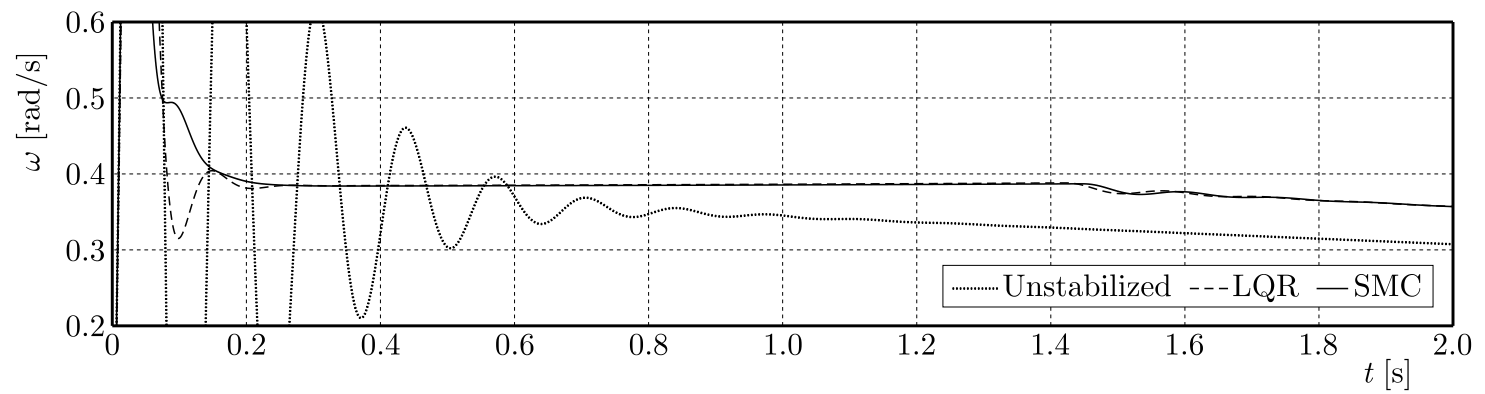

Fig. 7. Details of changes in the angular rates of the airframe

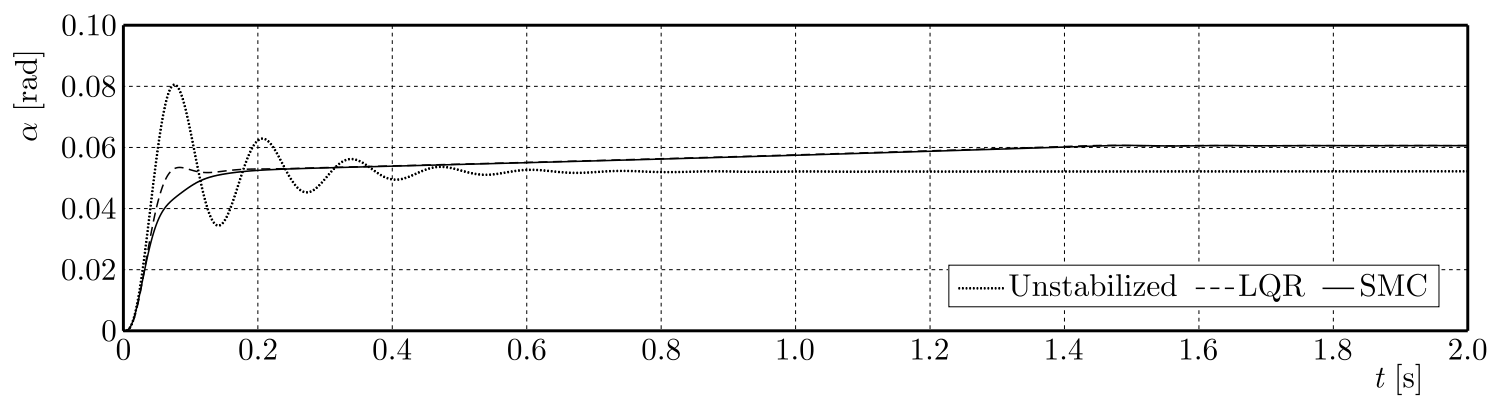

Fig. 8. Airframe angles of attack 


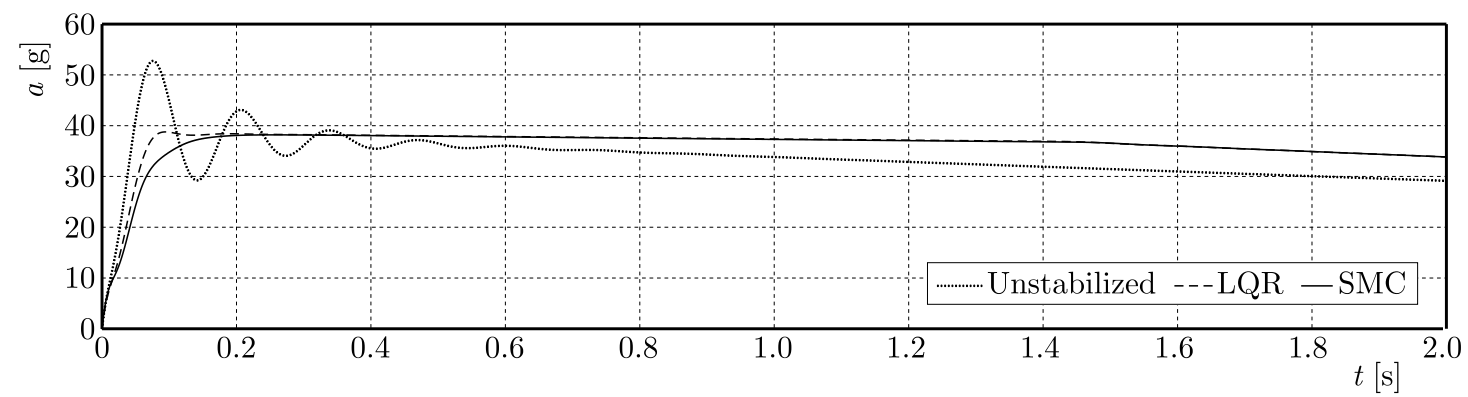

Fig. 9. Normal accelerations of the airframe

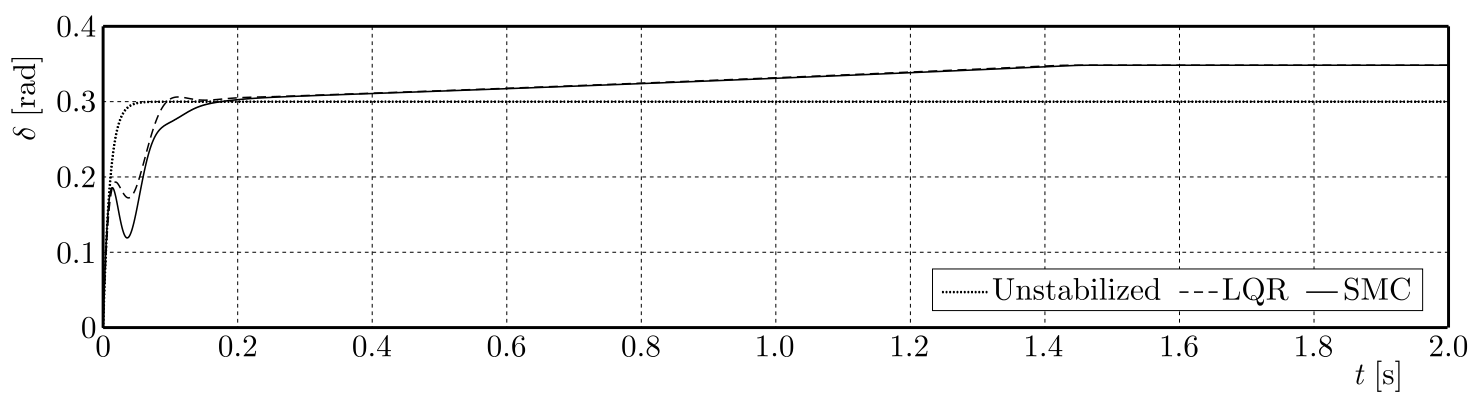

Fig. 10. Canard deflection angles

Table 2. Values of control quantity indices

\begin{tabular}{|c|c|c|}
\hline Airframe & $J_{\Omega}[-]$ & $J_{\Delta}[-]$ \\
\hline \hline Unstabilized & $2.015 \mathrm{e} 3$ & $2.955 \mathrm{e} 3$ \\
\hline LQR & $0.645 \mathrm{e} 3$ & $3.945 \mathrm{e} 3$ \\
\hline SMC & $0.611 \mathrm{e} 3$ & $4.768 \mathrm{e} 3$ \\
\hline
\end{tabular}

to decrease over time, in spite of the fact that the control signal $\kappa$ at the input to the system remains constant. The LQR and SMC stabilization systems aim to maintain a constant angular rate $\omega$, proportionally to the applied input signal $\kappa$ through manipulation of the canard deflection angle $\delta$ subject to the limitation imposed by the maximum deflection angle (Fig. 10). The stabilization system with a sliding mode controller is found to do this more effectively (Figs. 6 and 7), leading to the stabilization of changes in the angle of attack and normal acceleration of the airframe (Figs. 8 and 9). The drops in the airframe accelerations observed in Fig. 9 are related to a decrease over time in the velocity of the missile, caused by the drag force.

\section{Conclusions}

The SMC algorithm presented here is designed for implementation in systems for stabilizing static and dynamic characteristics of missiles - anti-aircraft missiles in particular. The algorithm effectively determines the input signal and provides effective separation from the dynamic characteristics of the airframe, which vary to a large degree during the flight.

It is possible to implement the algorithm in practice. To do so, one requires knowledge of the airframe angular rate $\omega$ and the angle of attack $\alpha$. The current angular rate in the control plane is supplied by a rate gyroscope. The angle of attack may be determined directly or indirectly: directly, by using an on-board instrument for measuring the angle of attack; indirectly, by means of Eq. (4.2) where the current pitch angle of the airframe $\vartheta$ is supplied by a free gyroscope, whereas the pitch angle of the velocity vector $\theta$ is obtained by integrating, with respect to time, the expression 


$$
\dot{\theta}=\frac{a}{V}
$$

where $a$ is the acceleration normal to the velocity vector $\mathbf{V}$, and $V$ is the modulus of that vector. The required values are obtained from accelerometers relative to the appropriate axes. Clearly, both approaches give rise to certain technical and implementation problems. Discussion of these issues is, however, beyond the scope of this paper. The results of the tests indicate that the use of the proposed stabilization system with the SMC algorithm in the autopilot of an anti-aircraft missile would improve the quality of control by stabilizing the angular rate of the airframe. The system transitional processes would also become shorter and smoother, leading to better operating conditions for the seeker installed in the missile and more effective guidance towards an aerial target.

\section{References}

1. Bużantowicz W., Pietrasieński J., 2018, Dual-control missile guidance: a simulation study, Journal of Theoretical and Applied Mechanics, 56, 3, 727-739

2. ÇImen T., 2008, State-dependent Riccati equation (SDRE) control: a survey, Proceedings of the 17th IFAC World Congress, 41, 2, 3761-3775

3. Erdem E.B., Alleyne A.G., 2004, Design of a class of nonlinear controllers via state dependent Riccati equations, IEEE Transactions on Control Systems Technology, 12, 1, 133-137

4. Grycewicz H., Mosiewicz R., Pietrasieński J., 1984, Radio Control Systems (in Polish), Military University of Technology, Warsaw

5. Gu W., Zhao H., Pan C., 2005, Sliding mode control for an aerodynamic missile based on backstepping design, Journal of Control Theory and Applications, 3, 1, 71-75

6. Idan M., Shima T., Golan O.M., 2007, Integrated sliding mode autopilot-guidance for dual control missiles, Journal of Guidance, Control, and Dynamics, 30, 4, 1081-1089

7. Kurow V.D., Do€żanski J.M., 1964, Principles behind the Solid-Propellant Rocket Design (in Polish), Publishing House of Ministry of National Defense, Warsaw

8. Shima T., Idan M., Golan O.M., 2006, Sliding-mode control for integrated missile autopilot guidance, Journal of Guidance, Control, and Dynamics, 29, 2, 250-260

9. Shtessel Y.B., Tournes C.H., 2009, Integrated higher-order sliding mode guidance and autopilot for dual-control missiles, Journal of Guidance, Control, and Dynamics, 32, 1, 79-94

10. Siddiq M.K., Cheng F.J., Bo Y.W., 2013, SDRE based integrated roll, yaw and pitch controller design for $122 \mathrm{~mm}$ artillery rocket, Applied Mechanics and Materials, 415, 200-208

11. Siouris G.M., 2004, Missile Guidance and Control Systems, Springer, New York

12. Yanushevsky R., 2007, Modern Missile Guidance, 1st Edition, CRC Press, New York

13. Zarchan P., 2012, Tactical and Strategic Missile Guidance, 6th Edition, AIAA, Reston

14. Zhou D., Mu C., Xu W., 1999, Adaptive sliding-mode guidance of a homing missile, Journal of Guidance, Control, and Dynamics, 22, 4, 589-594 